\title{
BLOQUEO ANGULAR AGUDO TRAS BRONCODILATADORES NEBULIZADOS
}

\section{ACUTE ANGLE-CLOSURE GLAUCOMA RESULTING FROM TREATMENT WITH NEBULISED BRONCHODILATORS}

\author{
FERNÁNDEZ-BARRIENTOS Y ${ }^{1}$, JIMÉNEZ-SANTOS M${ }^{1}$, MARTÍNEZ-DE-LA-CASA JM ${ }^{2}$, \\ MÉNDEZ-HERNÁNDEZ C ${ }^{2}$, GARCÍA-FEIJOÓ J
}

\begin{abstract}
RESUMEN
Caso clínico: Mujer de 78 años, con un cuadro de reagudización de enfermedad pulmonar obstructiva crónica tratada con bromuro de ipratropio y salbutamol nebulizados. Tras 20 horas desde el inicio del tratamiento, la paciente desarrolló un glaucoma agudo de ángulo cerrado (GACA) en el ojo izquierdo que respondió rápidamente al tratamiento médico adecuado.

Discusión: El bromuro de ipratropio y el salbutamol nebulizados, aumentan la presión intraocular y pueden desencadenar un GACA en pacientes predispuestos (cámara anterior estrecha, hipermétropes, glaucoma crónico de ángulo estrecho).

Una mayor precaución en los pacientes tratados con estos broncodilatadores, podría evitar este efecto adverso indeseable. Además, la correcta aplicación de la mascarilla facial, el uso de terminales en $\mathrm{T}$ y gafas de protección, son algunas medidas a tomar para prevenir un GACA en estos pacientes.
\end{abstract}

Palabras clave: Glaucoma agudo, glaucoma por cierre angular, insuficiencia respiratoria aguda, bromuro de ipratropio, salbutamol.

\begin{abstract}
Case report: A 78-year-old woman, with an acute exacerbation of chronic obstructive airways disease, was treated with nebulised ipratropium bromide and salbutamol. Twenty hours after beginning this treatment, she developed acute angle-closure glaucoma (AACG) in her left eye which resolved rapidly with appropriate treatment.

Discussion: Nebulised ipratropium bromide and salbutamol increases the intraocular pressure and may cause an AACG in susceptible patients (those with a shallow anterior chamber, hypermetropia, or chronic angle-closure glaucoma).

Increased vigilance in such patients treated with these bronchodilators may avoid this adverse effect. Ensuring the mask is correctly fitted, using a T-piece or unvented nebuliser and protective eye wear, are some of the many recommendations made to minimize the development of AACG in these patients (Arch Soc Esp Oftalmol 2006; 81: 657-660).
\end{abstract}

Key words: Acute glaucoma, angle-closure glaucoma, acute respiratory disease, ipratropium bromide, salbutamol.

\footnotetext{
Recibido: 8/2/06. Aceptado: 17/11/06

Hospital Clínico Universitario San Carlos. Instituto de Investigaciones Ramón Castroviejo. Madrid. España.

1 Licenciado en Medicina.

2 Doctor en Medicina.

Correspondencia:

Yolanda Fernández Barrientos

C/. Vallehermoso, $15,2^{\circ}$ apto. 20

28015 Madrid

España

E-mail: yferbar@terra.com
} 


\section{INTRODUCCIÓN}

Los broncodilatadores nebulizados (beta-agonistas y anticolinérgicos) son fármacos muy eficaces en el manejo de la insuficiencia respiratoria aguda en pacientes con enfermedad pulmonar obstructiva crónica (EPOC).

Se presenta un caso de glaucoma agudo por cierre angular (GACA) secundario a la administración de bromuro de ipratropio y salbutamol nebulizados.

\section{CASO CLÍNICO}

Mujer de 78 años, con EPOC en tratamiento con $50 \mu \mathrm{g}$ de salmeterol (Serevent accuhaler ${ }^{\circledR}$, Glaxo Smith Kline, Madrid, España) una inhalación cada 12 horas y $18 \mu \mathrm{g}$ de bromuro de tiotropio monohidrato (Spiriva ${ }^{\circledR}$, Boehringer Ingelheim Inc, Barcelona, España) una inhalación cada 24 horas, que acude a urgencias por reagudización de su patología respiratoria. Tras el estudio en la unidad de corta estancia, es diagnosticada una insuficiencia respiratoria aguda secundaria a neumonía, iniciándose tratamiento con oxigenoterapia, $250 \mu \mathrm{g}$ de bromuro de ipratropio (Atrovent ${ }^{\circledR}$ monodosis $250 \mu \mathrm{g}$; Boehringer Ingelheim Inc, Barcelona, España) y $2,5 \mathrm{mg}$ de salbutamol $\left(0,5 \mathrm{ml}\right.$ de Ventolin ${ }^{\circledR}$ solución para respirador; Glaxo Smith Kline, Madrid, España) mediante nebulizador con mascarilla facial cada 6 horas, 6-metilprednisolona (Urbason ${ }^{\circledR}$ soluble; Aventis Pharma, Madrid, España) $20 \mathrm{mg}$ cada 8 horas intravenoso, imipenem $500 \mathrm{mg} / 8$ horas IV y levofloxacino $500 \mathrm{mg} / 24$ horas IV.

A las 20 horas de su ingreso se solicita valoración a oftalmología por visión borrosa, dolor ocular leve y midriasis arreactiva del ojo izquierdo (OI). La paciente no tenía antecedentes oftalmológicos previos. A la exploración la agudeza visual era de 0,6 OD y 0,25 OI, en la biomicroscopía del polo anterior, se objetivó: hiperemia conjuntival moderada, midriasis arreactiva, cámara anterior muy estrecha con contacto iridoendotelial periférico y tyndall de $1+$. La presión intraocular (PIO) medida con tonómetro de aplanación era de $65 \mathrm{mmHg}$. El ángulo irido-corneal del ojo contralateral era estrecho (signo de Vena 0.2) y la PIO de $14 \mathrm{mmHg}$, la exploración del fondo de ojo sin dilatación no presentaba alteraciones significativas.

Se inició tratamiento con pilocarpina tópica al $2 \%$ (Colicursi pilocarpina ${ }^{\circledR} 2 \%$, Alcon Cusi SA, El Masnou, Barcelona, España) 1 gota cada 5 minutos durante 15 minutos, seguidamente 1 gota cada 15 minutos durante 1 hora y posteriormente 1 gota cada 6 horas, brimonidina $0,2 \%$ (Alphagan ${ }^{\circledR}$, Allergan SA, Tres Cantos, Madrid, España) 1 gota cada 12 horas, dexametasona fosfato sódico $1 \mathrm{mg} / \mathrm{ml}$ (Colorcusi dexametazona $^{\circledR}{ }^{\circledR}$, Alcon Cusi, SA, El Masnou, Barcelona, España) 1 gota cada 6 horas, acetazolamida $250 \mathrm{mg}$ vía oral $\left(\right.$ Edemox $^{\circledR}$, Chiesi Wasserman, Barcelona, España) $250 \mathrm{mg}$ cada 8 horas y Boi-K aspártico vía oral (BOI Laboratorios, Mollet del Valles, Barcelona, España). El bromuro de ipatropio y el salbutamol nebulizados fueron suspendidos. La presión intraocular descendió a 24 $\mathrm{mmHg}$ seis horas después del inicio de las medidas terapéuticas. Tras la realización de dos iridotomías periféricas con láser Nd: YAG en el OI la PIO era de $11 \mathrm{mmHg}$. En el ojo adelfo se realizó una iridotomía periférica profiláctica. Se mantuvo la brimonidina $0,2 \%$ cada $12 \mathrm{~h}$, la dexametasona cada 6 y se retiró el resto de la medicación tópica y oral.

A las 24 horas la cámara anterior del OI presentaba un signo de Vena de 0,4, que se correspondía con un ángulo grado II en la gonioscopia, las iridotomías eran permeables y el tyndall negativo. La presión intraocular era de $11 \mathrm{mmHg}$ OD y $8 \mathrm{mmHg}$ OI.

\section{DISCUSIÓN}

La EPOC es una enfermedad de gran prevalencia en nuestro medio. Afortunadamente, el GACA desencadenado por la medicación durante las reagudizaciones es una complicación poco frecuente.

En la tabla I se muestran los casos descritos en la literatura.

El bromuro de ipratropio es un agente anticolinérgico que induce midriasis, su mecanismo de acción a nivel ocular es por acción local, por depósito sobre la superficie conjuntival y corneal del fármaco nebulizado, frecuentemente por una mascarilla de inhalación mal ajustada. Parece que la absorción sistémica en el árbol traqueobronquial del bromuro de ipatropio es despreciable. El salbutamol es un agonista beta2-adrenérgico, además de provocar midriasis incrementa la producción de humor acuoso, sus efectos adversos a nivel ocular son por absorción local y sistémica $(1,2)$.

La midriasis media y el aumento del humor acuoso en pacientes predispuestos (cámara anterior estrecha, hipermétropes), puede desencadenar un cierre angular agudo por bloqueo pupilar. La mayo- 
Tabla I. Resumen de los casos publicados hasta la fecha

\begin{tabular}{|c|c|c|c|c|}
\hline & N..$^{o}$ de casos & Fármacos & $\begin{array}{l}\text { Inicio de los síntomas } \\
\text { oculares }\end{array}$ & $\begin{array}{l}\text { Antecedentes } \\
\text { oculares }\end{array}$ \\
\hline Malani JT et al & 2 & $\mathrm{BI}^{*}$ & & \\
\hline Holst PE et al & 1 & BI & & \\
\hline Packe GE et al & 1 & $\mathrm{BI}+\mathrm{S} \dagger$ & 24 horas & \\
\hline Shah P et al & 5 & $\mathrm{BI}+\mathrm{S}$ & 24 horas $\pm 78,5$ horas & \\
\hline Reuser $\mathrm{T}$ et al & 2 & $\mathrm{BI}+\mathrm{S}$ & 3 días \pm Odías & Hipermétrope \\
\hline Mulpeter KM et al & 3 & $2 \mathrm{BI}+\mathrm{S}$ & & \\
\hline $1 \mathrm{BI}$ (inhalado + nebulizado) & 6 días $\pm 0,94$ días & & & \\
\hline Hall SK & 1 & BI (inhalado)+S & 36 horas & GACA \\
\hline Le Llouche $\mathrm{N}$ et al & 1 & $\mathrm{BI}+\mathrm{S}$ & 24 horas & hipermétrope \\
\hline Rho DS et al & 1 & S (nebulizado + inhalado) & 1 hora & \\
\hline De Saint Jean M & 1 & $\mathrm{~S}+\mathrm{BI}$ & 24horas & \\
\hline Boehringer Ingelheim & 4 & BI & & \\
\hline
\end{tabular}

* BI: bromuro de ipatropio; $\uparrow \mathrm{S}$ : salbutamol.

ría de los casos descritos en la literatura no presentaban antecedentes de glaucoma (tabla I).

El agente más importante implicado en este cuadro es el bromuro de ipratropio, aunque también se han descrito casos de GACA desencadenados sólo por el salbutamol. La incorrecta aplicación de la mascarilla facial y las elevadas dosis farmacológicas de estos fármacos en nebulización facilitan el desarrollo del GACA $(1,2)$.

Karla y Bone, describieron los efectos de la administración conjunta de bromuro de ipratropio y salbutamol en pacientes con glaucoma y bronquitis crónica. La PIO aumentó sólo en pacientes con GAE, de éstos, la mitad desarrollaron un cierre angular agudo transitorio. Los pacientes que recibieron la medicación conjunta y llevaban gafas de piscina como protección ocular, no mostraron cambios en la PIO ni en el ángulo, demostrando que la acción del fármaco era por un depósito directo en el saco conjuntival y no por absorción sistémica (3).

El desarrollo de GACA es más frecuente con la combinación bromuro de ipratropio y salbutamol, versus su administración aislada. Esta asociación es segura, se emplea en un $77 \%$ de los pacientes con reagudización de EPOC, aunque no hay estudios que sugieran su superioridad frente al uso individual (4).

Cuando la dosificación del tratamiento sea en nebulización con mascarilla facial, ésta ha de ajustarse correctamente y se pueden usar gafas de piscina protectoras en pacientes de riesgo. El uso de terminales de tubo en $\mathrm{T}$, reduce la exposición ocular al fármaco. En algunos casos, en cuanto el paciente esté estable se podrán utilizar inhaladores de dosis medidas (IDM) con cámara espaciadora (5).

Una mejor detección de los pacientes en riesgo de desarrollar un bloqueo angular, evitaría este efecto adverso. Además, la corrrecta aplicación de la mascarilla facial, el uso de terminales en $\mathrm{T}$ y de IDM con cámara espaciadora, reducen la exposición ocular al fármaco. Los pacientes con mayor riesgo de GACA en los que sea necesario el tratamiento nebulizado podrían utilizar gafas de piscina como protección, medicación hipotensora ocular preventiva y/o iridotomía con láser YAG.

\section{BIBLIOGRAFÍA}

1. Hall SK. Acute angle-closure glaucoma as a complication of combined beta-agonist and ipratropium bromide therapy in the emergency departament. Ann Emerg Med 1994; 23: 884-887.

2. Rho DS. Acute angle-closure glaucoma after albuterol nebuliser treatment. Am J Ophthalmol 2000; 130: 123-124.

3. Kalra L, Bone MF. The effect of nebulized bronchodilator therapy on intraocular pressures in patients with glaucoma. Chest 1988; 93: 739-741.

4. McCrory DC, Brown CD. Broncodilatadores anticolinérgicos versus agentes beta2simpaticomiméticos para las exacerbaciones agudas de la enfermedad pulmonar obstructiva crónica (Revisión de Cochrane traducida). En: La Biblioteca Cochrane Plus, 2005, Número 4. Oxford: Update Software Ltd. Disponible en: http://www.updatesoftware.com

5. Cates CJ, Bara A, Crilly JA, Rowe BH. Cámaras espaciadoras versus nebulizadores para el tratamiento del asma aguda con betaagonistas (Revisión Cochrane traducida). En: La Biblioteca Cochrane Plus, 2005. Número 4. Oxford: Update Software Ltd. Disponible en: http://www.update-software.com 